\title{
Nonredundant roles of antibody, cytokines, and perforin in the eradication of established Her-2/neu carcinomas
}

\author{
Claudia Curcio, ${ }^{1}$ Emma Di Carlo, ${ }^{2}$ Raphael Clynes, ${ }^{3}$ Mark J. Smyth, ${ }^{4}$ Katia Boggio, ${ }^{1}$ \\ Elena Quaglino, ${ }^{1}$ Michela Spadaro, ${ }^{1}$ Mario P. Colombo, ${ }^{5}$ Augusto Amici, ${ }^{6}$ \\ Pier-Luigi Lollini, ${ }^{7}$ Piero Musiani, ${ }^{2}$ and Guido Forni ${ }^{1,8}$ \\ ${ }^{1}$ Department of Clinical and Biological Sciences, University of Turin, Orbassano, Italy \\ ${ }^{2}$ Department of Oncology and Neuroscience, G. d'Annunzio University, Chieti, Italy \\ ${ }^{3}$ Department of Medicine and Microbiology, Columbia University College of Physicians and Surgeons, \\ New York, New York, USA \\ ${ }^{4}$ Department of Cancer Immunology, Peter MacCallum Institute, East Melbourne, Victoria, Australia \\ ${ }^{5}$ Division of Experimental Oncology, National Tumor Institute, Milan, Italy \\ ${ }^{6}$ Department of Molecular, Cellular, and Animal Biology, University of Camerino, Camerino, Italy \\ ${ }^{7}$ Cancer Research Section, Department of Experimental Pathology, University of Bologna, Bologna, Italy \\ ${ }^{8}$ Center for Experimental Research and Medical Studies, Ospedale San Giovanni Battista, Turin, Italy
}

\begin{abstract}
Since the mechanisms by which specific immunity destroys Her-2/neu carcinoma cells are highly undetermined, these were assessed in $\mathrm{BALB} / \mathrm{c}$ mice vaccinated with plasmids encoding extracellular and transmembrane domains of the protein product ( $\left.\mathrm{p} 185^{\text {neu}}\right)$ of the rat Her-2/neu oncogene shot into the skin by gene gun. Vaccinated mice rejected a lethal challenge of TUBO carcinoma cells expressing $\mathrm{p}^{185^{\text {neu }}}$. Depletion of CD4 T cells during immunization abolished the protection, while depletion of $\mathrm{CD} 8$ cells during the effector phase halved it, and depletion of polymorphonuclear granulocytes abolished all protection. By contrast, Ig $\mu$-chain gene $\mathrm{KO}$ mice, as well as Fc $\gamma$ receptor I/III, $\beta$-2 microglobulin, CD1, monocyte chemoattractant protein 1 (MCP1), IFN- $\gamma$, and perforin gene KO mice were protected. Only mice with both IFN- $\gamma$ and perforin gene KOs were not protected. Although immunization also cured all $\mathrm{BALB} / \mathrm{c}$ mice bearing established TUBO carcinomas, it did not cure any of the perforin $\mathrm{KO}$ or perforin and IFN- $\gamma \mathrm{KO}$ mice. Few mice were cured that had knockouts of the gene for Ig $\mu$-chain, Fc $\gamma$ receptor I/III, IFN- $\gamma$, or $\beta-2$ microglobulin. Moreover, vaccination cured half of the CD1 and the majority of the MCP1 KO mice. The eradication of established $\mathrm{p} 185^{\text {neu }}$ carcinomas involves distinct mechanisms, each endowed with a different curative potential.
\end{abstract}

J. Clin. Invest. 111:1161-1170 (2003). doi:10.1172/JCI200317426.

\section{Introduction}

Many experimental and human tumors express tumorassociated antigens (TAAs) that are recognized by $\mathrm{T}$ cells (1) and antibodies (2). The role of T lymphocytes in reacting against TAAs in the eradication of established tumors (3) is widely documented. In most cases, tumor inhibition rests on the direct lytic activity of cytotoxic CD8 T lymphocytes (1-4), whereas CD4 T cells activated through the indirect presentation of TAAs by antigen-presenting cells (APCs) are required to support a CD8 $\mathrm{T}$ cell response

Received for publication November 19, 2002, and accepted in revised form January 30, 2003.

Address correspondence to: Guido Forni, Department of Clinical and Biological Sciences, University of Turin, 10043 Orbassano, Italy. Phone: 39-011-670-8119; Fax: 39-011-903-8639; E-mail: guido.forni@unito.it.

Conflict of interest: The authors have declared that no conflict of interest exists.

Nonstandard abbreviations used: tumor-associated antigen (TAA); antigen-presenting cell (APC); Fc $\gamma$ receptor $(\mathrm{Fc} \gamma \mathrm{R})$; antibody-dependent cellular cytotoxicity (ADCC); perforin (pfp); polymorphonuclear cell (PMN).
$(5,6)$. Tumor eradication also depends on noncytotoxic CD4 (7) and CD8 (6) lymphocytes releasing high amounts of proinflammatory cytokines that recruit distinct effector leukocytes at the tumor site. This series of events gives rise to a complex delayed-type hypersensitivity reaction in the tumor site that involves several distinct cell types (8).

Most experimental evidence supports the notion that tumor eradication rests predominantly, if not exclusively, on T lymphocyte reactivity (9). However, the importance of antibody in the inhibition of solid tumors is not clearly defined and remains controversial. Some experimental data have shown that antibodies might enhance tumor growth by hindering the cell-mediated mechanisms on which tumor inhibition may depend $(10,11)$. Spontaneous anti-TAA antibodies are frequently detected in the serum of cancer patients $(2,12,13)$. Although their titer may increase with the progression of the tumor (14), this rise actually correlates with a poor prognosis (15). Moreover, the presence of $\mathrm{B}$ cells and a nonprotective antitumor humoral immune response may inhibit the induction of protective $T$ cell-dependent antitumor immunity (16). 
On the other hand, the enhanced TAA cross-presentation that takes place in the presence of antibodies (17), as well as the binding of anti-TAA antibodies to the $\mathrm{Fc} \gamma$ receptors ( $\mathrm{Fc} \gamma \mathrm{Rs}$ ) on APC surfaces (18), may result in the eradication of established tumors in synergy with CD8 T cells (19). In addition, antibodies can sensitize tumors for complement and antibodydependent cytotoxicity, or they may activate antibodydependent cellular cytotoxicity (ADCC) through their binding to Fc $\gamma$ R on leukocyte membrane $(20,21)$. Furthermore, the results of several vaccination trials have shown that improved survival correlates with the induction of antibodies to TAA $(22,23)$, and noticeable inhibitions of tumor progression can be obtained through the passive administration of $\mathrm{mAb}(24,25)$.

Besides the activation of immunological functions, antibodies directed against oncogenic tyrosine kinase receptors directly impair the proliferative activity of the target cells. Among these receptors, the protein product of the Her-2/neu oncogene ( $\mathrm{p} 185^{\text {neu}}$ ) has been studied extensively. Antibodies may induce a $185^{\text {neu }}$ functional block (26), downregulate its expression on the cell membrane, and impede its ability to form the homo or heterodimers that spontaneously transduce proliferative signals to the cells (26-28). Antibodies hamper the three-dimensional growth of Her-2/neu mouse mammary carcinoma cells (29) and play an important role in delaying the onset of mammary carcinomas in Her-2/neu transgenic mice (27, 30-32). However, the importance of reaction mechanisms dependent on anti-p185 18 antibodies in the resistance to a $185^{\text {neu }}$ tumor is controversial. Although in a few systems antibodies appear to play an important role in the rejection of $\mathrm{p} 185^{\text {neu }}$ tumors $(33,34)$, in others $\mathrm{T}$ cell reactivity appears to be sufficient for tumor rejection (35-38).

In this study, the relative contribution of antibodymediated reactivity and cell-mediated reactivity was assessed in the rejection of a lethal challenge and the eradication of an established carcinoma formed by a transplantable cell line (TUBO cells) expressing rat $\mathrm{p} 185^{\text {neu }}$ $\left(\mathrm{r}-\mathrm{p} 185^{\mathrm{neu}}\right)(30)$. In this experimental system, $\mathrm{r}-\mathrm{p} 185^{\text {neu }}$ is both a xenogeneic surrogate TAA that differs from mouse p $185^{\text {neu }}$ in less than $6 \%$ of the amino acid residues (39) and a membrane receptor that directly regulates the oncogenic behavior of TUBO cells in vitro and in vivo (29, 30). WT and various immunodeficient BALB/c mice were immunized with DNA plasmids coding for the extracellular and transmembrane domains of r-p185 ${ }^{\text {neu }}$ (p185 plasmids) (30) shot into the skin by a "gene gun" (40). Gene gun DNA immunization is an efficient way to elicit tumor immunity through antigen cross-presentation (40-43). After gene gun vaccination, distinct cellular and humoral immune mechanisms independently led to the rejection of the lethal TUBO cell challenge. By contrast, only the combination of several cellular and humoral mechanisms led to the eradication of established tumors.

\section{Methods}

Mice. In these experiments, 7-week-old virgin female WT and gene $\mathrm{KO} \mathrm{BALB} / \mathrm{c}$ mice $\left(\mathrm{H}-2^{\mathrm{d}}\right)$ were used. WT
$\mathrm{BALB} / \mathrm{cnAnCr}(\mathrm{BALB} / \mathrm{c})$ mice were from Charles River Laboratories (Calco, Italy); mice with the Ig $\mu$ heavy-chain gene $\mathrm{KO}(16)$ (BALB- $\mu$ IgKO) were kindly provided by Thomas Blankenstein (Freie University, Berlin, Germany); mice with the FcyRI/III gene KO (44) (BALBFc $\gamma$ RI/IIIKO) were from Taconic Laboratories (Germantown, New York, USA); mice with the $\beta 2$-microglobulin gene $\mathrm{KO}$ (BALB- $\beta 2 \mathrm{mKO}$ ) (45) and the IFN- $\gamma$ gene $\mathrm{KO}$ (BALB-IFN $\gamma \mathrm{KO}$ ) (46) were from Jackson Laboratories (Bar Harbor, Maine, USA); mice with the CD1 gene KO (47) (BALB-CD1KO) were kindly provided by Luc Van Kaer (Vanderbilt University, Nashville, Tennessee, USA); mice with the MCP-1 gene KO (48) were kindly provided by Barrett Rollins (Dana Farber Institute, Boston, Massachusetts, USA); mice with the perforin (pfp) gene KO (BALB-pfpKO) and the IFN- $\gamma$ gene double KO (49) (BALB-IFN $\gamma$-pfpKO) were from the Peter MacCallum Cancer Institute (East Melbourne, Australia). Mice were treated properly and humanely in accordance with European Community guidelines. When required, 22, 18, 14, 8 , and 5 days before tumor challenge (day 0 ) or $2,5,8,11$, and 13 days after, a few mice received intraperitoneal injections of $0.2 \mathrm{ml}$ of HBSS containing a 1:20 dilution of antiasialo GM1 rabbit anti-serum (Wako Chemicals $\mathrm{GmbH}$, Dusseldorf, Germany) or $200 \mu \mathrm{g}$ of anti-CD4 (GK1.5 hybridoma, L3T4, American Type Culture Collection, Rockville, Maryland, USA), anti-CD8 (TIB-105 hybridoma, Lyt 2 ATCC), or anti-polymorphonuclear cell (PMN) mAb (RB6-8C5 hybridoma kindly provided by R.L. Coffman, DNAX Inc., Palo Alto, California, USA). Flow cytometry of residual blood and spleen cells collected 3 days after the last injection showed that target leukocytes were selectively decreased to below 1 per 5000 cells.

Cell lines. TUBO cells (30) are a cloned line derived from a BALB/c mice transgenic for the transforming $r$-Her-2/ neu oncogene (BALB-NeuT). N202.1A and N202.1E (32) are cloned cell lines derived from a FVB mice (H-2q) transgenic for the $r$-Her-2/neu proto-oncogene (FVB-NeuN) (32). F1F is a newborn WT BALB/c mouse-derived skin fibroblast line spontaneously transformed after the 15 th in vitro passage (6). F1F were transfected with the fulllength r-Her-2/neu gene (F1F-neu) cloned into pCDNA3 (Invitrogen, San Giuliano Milanese, Italy) or transduced with the $\beta$-galactosidase gene (F1- $\beta$ gal) by means of the LBSN retroviral vector as described previously (50). r-p185 ${ }^{\text {neu }}$ is highly expressed on the cell membrane of TUBO (30), N202.1A (32), and F1F-neu cells (data not shown), but it is absent on N202.1E (32), TSA mammary carcinoma cell line (30), F1F (30), and F1F- $\beta$ gal (data not shown) cell surfaces. $\mathrm{H}-2 \mathrm{D}^{\mathrm{d}}$ and $\mathrm{H}-2 \mathrm{D}^{\mathrm{q}}$ glycoproteins are expressed by all BALB/c and FVB tumors, respectively (30, 32), but no cell lines expressed $\mathrm{H}-2$ class II glycoproteins (data not shown). N202.1A, N202.1E, TSA, F1F, F1F- $\beta$ gal, and F1F-neu cells were cultured in RPMI 1640 medium supplemented with $10 \%$ FBS, and TUBO cells were cultured in DMEM with $20 \%$ FBS. Neomycin $(800 \mu \mathrm{g} / \mathrm{ml})$ was added to the culture medium of F1F$\beta$ gal and F1F-neu cells. Media were from BioWhittaker Europe (Verviers, Belgium), FBS was from Life 
Technologies (San Giuliano Milanese, Italy), and neomycin was from Life Technologies (Milan, Italy).

Tumor challenge. Mice were challenged subcutaneously in the right flank with $0.2 \mathrm{ml}$ of a single suspension containing the minimal lethal dose of TUBO $\left(10^{5}\right)(30)$, F1F $\left(10^{4}\right)(6)$, F1F- $\beta$ gal $\left(10^{6}\right)$, and F1F-neu $\left(5 \times 10^{3}\right)$ cells. The cages were coded, and the incidence and the growth of tumors were evaluated twice weekly in a blind fashion. Neoplastic masses were measured with calipers in the two perpendicular diameters, and the mean diameter was recorded for 200 days. At the end of this period, tumor-free mice were classed as survivors. Mice were killed for humane reasons when the tumor exceeded $10 \mathrm{~mm}$ in mean diameter.

DNA expression vectors and vaccination. The $\mathrm{PCMV}$ vector was derived from the pcDNA3 plasmid (Invitrogen, San Diego, California, USA) by deleting the SV40 promoter, the neomycin resistance gene, and SV40 poly(A). The sequence for the extracellular and transmembrane domains of mutated r-p185 neu were generated from the PCR product using primers 3'-CGCAAGCTTCATCATGGAGCTGGC-5' and '3'-CGGAATTCGGGCTGGCTCTCTGCTC- $5^{\prime}$ and primers $3^{\prime}$-CGCAAGCTTCATGGAGCTGGC- $5^{\prime}$ and 3 '-ATGAATTCTTTCCGCATCGTGTACTTCTTCCGG-5', respectively, as previously described in detail (30). PCR products of the expected size were isolated by agarose gel electrophoresis, digested with HindIII and EcoRI, and cloned into the multiple cloning site of the PCMV plasmid to obtain the plasmid used in this work (p185 plasmid). Escherichia coli strain DH5 $\alpha$ was transformed with p185 plasmid and then grown in Luria-Bertani medium (Sigma-Aldrich, St. Louis, Missouri, USA). Large-scale preparation of the plasmid was conducted by alkaline lysis using Endofree Quiagen Plasmid-Giga kits (Quiagen, Chatsworth, California, USA). DNA was then precipitated, suspended in sterile saline at a concentration of $1 \mathrm{mg} / \mathrm{ml}$, and stored in aliquots at $-20^{\circ} \mathrm{C}$ for subsequent use in immunization protocols.

Gene gun plasmid administration. Mice were vaccinated with empty plasmids or p185 plasmids shot intradermally by a hand-held Helios Gene Gun system (Biorad, Hercules, California, USA) (40). Plasmids were precipitated onto 1.6- $\mu \mathrm{m}$ gold particles. Coated particles were then washed in absolute ethanol and inserted into a Tefzel tube (Biorad) that was cut into 0.5 -inch cartridges. In this way, $0.25 \mathrm{mg}$ of gold coated with $0.465 \mu \mathrm{g}$ of plasmid DNA was administered by each shot cartridge. Each vaccination consisted of three shots $(2.79 \mu \mathrm{g}$ of plasmid DNA per shot) administered with a 400-psi helium gas pulse in three distinct areas of shaved abdominal skin. In prevention experiments, mice were vaccinated on days 21 and 7 before tumor challenge (day 0). In other experiments, mice were first vaccinated when they presented a palpable tumor mass of 2,4 , or $6 \mathrm{~mm}$ in mean diameter and were boosted 7 days later.

Cellular cytotoxicity. CTLs were generated by culturing $10^{7}$ responder lymphocytes and $5 \times 10^{5}$ stimulator TUBO cells pretreated with $40 \mathrm{mg} / \mathrm{ml}$ of mitomycin-C for 40 minutes for 6 days (6). Cytotoxicity was assayed in a 48-hour $\left[{ }^{3} \mathrm{H}\right]$ thymidine release assay (30) by mixing various concentrations of effector cells with $5 \times 10^{3}$ labeled target cells at 50:1, 25:1, 12:1, and 6:1 effector/target ratios in round-bottom 96-well microtiter plates in triplicate as described previously. The values were expressed as the percentage of specific lysis (6).

Cytofluorometric evaluation of anti-r-p185nen antibodies. Sera of six WT BALB/c mice shot with empty vector or p185 plasmid were collected 2 weeks after the first vaccination and pooled. The ability of sera to bind $\mathrm{r}$ p185 neu was evaluated by flow cytometry. N202.1A or $\mathrm{N} 202.1 \mathrm{E}$ cells $\left(2 \times 10^{5}\right)$ from in vitro cultures were washed twice with cold PBS supplemented with $2 \%$ BSA and $0.05 \%$ sodium azide and stained in a standard indirect immunofluorescence procedure with $50 \mu \mathrm{l}$ of a $1: 10$ dilution of control or immune sera in PBS-azide-BSA. A fluorescein-conjugated rabbit anti-mouse Ig (Dako, Glostrup, Denmark) was used as a second step Ab. The cells were resuspended in PBS-azide-BSA containing 1 $\mathrm{mg} / \mathrm{ml}$ of propidium iodide to gate out dead cells and evaluated with a FACScan (Becton Dickinson and Co., Mountain View, California, USA). The specific N202.1A binding potential of the sera was calculated according to the following equation: ([\% positive cells with test serum][fluorescence mean]) $\times$ serum dilution. A total of $5 \times 10^{3}$ viable cells were analyzed in each evaluation.

Morphological analysis. Groups of three WT BALB/c and $\mathrm{KO}$ mice were sacrificed at the following times: WT $\mathrm{BALB} / \mathrm{c}$ mice vaccinated before tumor cell challenge were sacrificed 3 and 7 days after the last shot, and groups of WT BALB/c and KO mice shot when the tumor reach 2 $\mathrm{mm}$ in mean diameter were sacrificed each week beginning the second week after the first shot until the end of the experiment. For histological evaluation, tissue samples were fixed in $10 \%$ neutral buffered formalin, embedded in paraffin, sectioned at $4 \mu \mathrm{m}$, and stained with hematoxylin and eosin or Giemsa and the trichrome method. For immunohistochemistry, acetone-fixed cryostat sections were incubated for 30 minutes with antidendritic cells (NLDC 145; Cederlane, Hornby, Ontario, Canada), antiCD4, anti-CD8a (both from Sera-Lab, Crawley Down, Sussex, United Kingdom), anti-Mac-1 (anti-CD11b/ CD18), anti-Mac-3, anti-Ia (all from Boehringer Mannheim, Milan, Italy), anti-PMN leukocytes (RB6-8C5, provided by R.L. Coffman), antiasialo GM1 (Wako Chemicals $\mathrm{GmbH}$ ), antiendothelial cells (mEC-13.324), anti-ELAM1 (E selectin; both provided by A. Vecchi, Istituto M. Negri, Milan, Italy), anti-ICAM-1 (CD54), anti-VCAM-1, anti-IL-4, anti-IL-6 (PharMingen, San Diego, California, USA), anti-IL-1 $\beta$ (Genzyme, Cambridge, Massachusetts, USA), anti-TNF- $\alpha$ (Immuno Kontact, Frankfurt, Germany), anti-IFN- $\gamma$ (provided by S. Landolfo, University of Turin), and TGF- $\beta 1$ (Santa Cruz Biotechnology, Santa Cruz, California, USA) Ab's. To evaluate the expression of r-p185 $5^{\text {neu }}$, paraffin-embedded sections were tested with anti-p185 ${ }^{\text {neu }}$ antibodies (C-18-G, Santa Cruz Biotechnology). After washing, sections were overlaid with biotinylated goat anti-rat, anti-hamster, and anti-rabbit or horse anti-goat Ig (Vector Laboratories, Burlingame, California, 
Table 1

Specificity of the reaction elicited by Her-2/neu plasmids shot into WT BALB/c mice by gene gun

Tumor takes per mouse after a challenge with the indicated cell line:

Immunization with $\mathrm{BALB} / \mathrm{c}$ mice:

$$
\text { TUBO (p185 } 5^{\text {neu }} \text { positive) }
$$

$10 / 10(0 \%)$

$0 / 16(100 \%)$
F1F ( $185^{\text {neu }}$ negative)
ND
6/6 (0\%)
F1F- $\beta$ gal ( $p 185^{\text {neu }}$ negative)
ND
$5 / 5(0 \%)$

\author{
F1F-neu ( $185^{\text {neu }}$ positive) \\ $5 / 5(0 \%)$ \\ $0 / 5(100 \%)$
}

WT BALB/c mice were immunized twice with p185 plasmid or empty vector 21 and 7 days before tumor challenge (day 0 ) with tumor cells expressing (TUBO and F1F-neu) or not expressing (F1F and F1F- $\beta$ gal) p185neu. Tumor takes and percentage of protected mice were evaluated for 100 days after challenge. A significant protection was observed against TUBO and F1F-neu cells in mice vaccinated with p185 plasmids $\left(P<0.001\right.$, Yates's corrected $\chi^{2}$ test). ND, not done.

USA) for 30 minutes. Unbound antibody was removed by washing, and the slides were incubated with $\mathrm{ABC}$ complex/AP (Dako). Quantitative studies of immunohistochemically stained sections were performed independently by three pathologists in a blinded fashion. From mice with multiple tumors, one sample per tumor growth area and 10 randomly chosen fields in each sample were evaluated for each point determination. Positive cells were counted under a $\times 400$-field microscope $(x 40$ objective and $\times 10$ ocular lens, $0.180 \mathrm{~mm}^{2}$ per field). The expression of adhesion molecules, cytokines, and mediators was defined as absent $(-)$ or scarcely $( \pm)$, moderately $(+)$, or frequently $(++)$ present on cryostat sections tested with the corresponding $\mathrm{Ab}(30,32)$.

Statistical analysis. Differences in tumor takes were evaluated by Yates' corrected $\chi^{2}$ test, and differences in the number of tumor-infiltrating cells were evaluated by the two-tailed Student's $t$ test.

\section{Results}

$W T B A L B / c$ mice do not react against $r$ - $p 185^{\text {neu }}$ expressed by tumor cells. In mice, $\mathrm{r}-\mathrm{p} 185^{\text {neu }}$ is a xenogeneic surrogate TAA, since it differs from mouse $185^{\text {neu }}$ in less than $6 \%$ of the amino residues (39). Despite this difference, the TUBO and F1F-neu cell challenges grew progressively in all WT BALB/c mice (Table 1), and their growth kinetics were not different from those observed in transgenic BALB-NeuT mice, from which TUBO cells were derived (30). During TUBO and F1F-neu tumor growth, no anti-r-p185 ${ }^{\text {neu }}$ antibodies were detectable in the sera of WT BALB/c mice, nor were CTL activity or cytokine release found when splenocytes from TUBOand F1F-neu-bearing WT BALB/c mice were restimulated in vitro with TUBO cells (30) (data not shown). In addition, the reactive cell infiltrate associated with the growth of TUBO and F1F-neu tumors in both WT $\mathrm{BALB} / \mathrm{c}$ and BALB-NeuT mice was equally marginal and not significantly different from that associated with the fully syngeneic F1F and TSA tumors (data not shown).

Gene gun DNA vaccination protects WT BALB/c mice against an $r$-p185neu tumor challenge. Despite the ostensible immunological invisibility of r-p185 18 expressed by proliferating tumor cells, both TUBO and F1F-neu tumor cell challenges were specifically rejected by WT $\mathrm{BALB} / \mathrm{c}$ mice preimmunized with $\mathrm{p} 185$ plasmids administered 21 and 7 days before challenge (Table 1 ).
TUBO- and F1F-neu tumor cells first formed small aggregates that were quickly and massively infiltrated by reactive leukocytes consisting of dendritic cells, macrophages, PMNs, CD8 and $\mathrm{CD}^{+}$lymphocytes, and NK cells. The prevalence of CD8 lymphocytes and PMNs was about fivefold that of TUBO tumors growing in untreated mice. In addition, a relevant expression of endothelial adhesion molecules (ICAM-1, ELAM-1, and VCAM- 1 ) and cytokines (IL- $1 \beta$, TNF- $\alpha$, IFN- $\gamma$, and IL-4) was observed. Expression of r-p185 neu in tumor cells was mainly confined to the cell cytoplasm (data not shown).
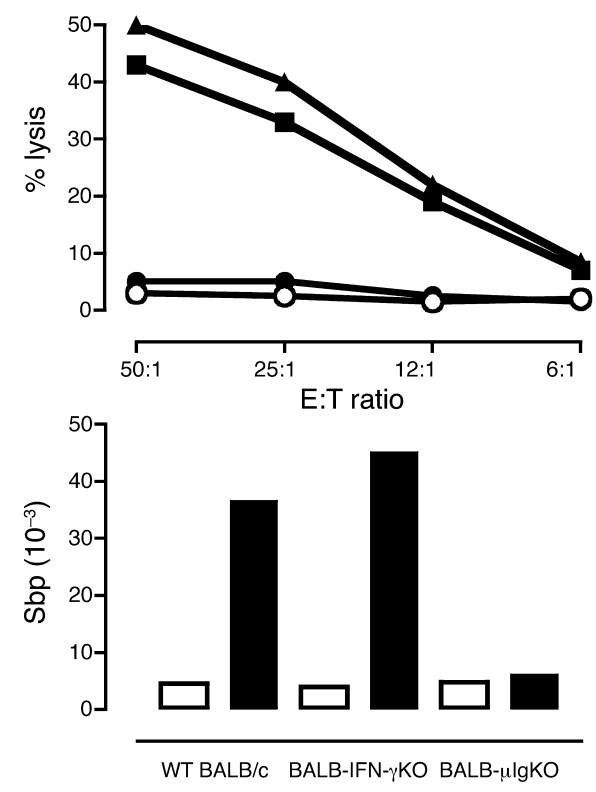

\section{Figure 1}

Cytotoxic and antibody response to $\mathrm{p} 185^{\text {neu }}$ of WT BALB/c mice shot at 2 week intervals with $\mathrm{p} 185$ plasmids. Spleens and sera were collected 2 weeks after the last immunization. The upper panel shows cytotoxicity of splenocytes evaluated in a 48 -hour $\left[{ }^{3} \mathrm{H}\right]$ thymidine release assay against TUBO (squares, $\mathrm{H}-2^{\mathrm{d}}, \mathrm{p} 185^{\text {neu }}$ positive), F1F-neu (triangles, $\mathrm{H}-2^{\mathrm{d}}$, $\mathrm{P} 185^{\text {neu }}$ positive), N202.1A (filled circles, $\mathrm{H}-2^{\mathrm{q}}, \mathrm{P} 185^{\text {neu }}$ positive); and F1F (open circles, $\mathrm{H}-2^{\mathrm{d}}, \mathrm{p} 185^{\text {neu }}$ negative) targets. No significant cytotoxicity was found in mice shot with empty plasmids (data not shown). The lower panel shows the specific binding potential titer of anti-p185 neu $A b$ in a pool of sera from WT BALB/c, BALB-IFN $\gamma K O$, and BALB- $\mu$ IgKO mice shot with empty (white bars) or 185 (black bars) plasmids. 
Vaccination with p185 plasmids elicited both significant cytolytic activity restricted to r-p $185^{\text {neu }} \mathrm{H}-2 \mathrm{D}^{\mathrm{d}}$ target cells (Figure 1, upper panel) and high titers of antir-p185 ${ }^{\text {neu }}$ antibodies (Figure 1, lower panel). The memory elicited was still able to protect all mice when the TUBO cell challenge was performed 140 days after the second plasmid administration by gene gun (data not shown). Immune mechanisms leading to the rejection of an $r-p 185^{\text {neu }}$ tumor challenge. To assess the relative weight of immune mechanisms elicited by gene gun vaccination, both WT $\mathrm{BALB} / \mathrm{c}$ mice with depletions in selected leukocyte populations and $\mathrm{BALB} / \mathrm{c}$ mice with null mutations in genes regulating immunological functions were challenged with TUBO cells (Table 2). The depletion of CD4 cells during the induction of the response, but not during the effector phase, abolished any capacity to reject the tumor challenge. Similarly, the removal of CD8 lymphocytes and PMNs during the effector phase (though not during the immunization) strongly impaired the capacity of immunized mice to reject TUBO cells. These selected leukocyte depletions did not influence TUBO cell growth in nonimmunized mice (data not shown). Irrespective of the missing gene, TUBO cells grew out in all nonimmunized $\mathrm{BALB} / \mathrm{c} \mathrm{KO}$ mice but were rejected by all mice that had been immunized. Only in mice lacking both functioning pfp and IFN- $\gamma$ genes and in mice with depletion of PMNs was there a complete failure to elicit protective immunity. In immunized mice, PMN antitumor activity can be triggered and guided by cytokines released by $T$ cells and antibody $(20,51)$. Even when immunized mice were challenged with larger amounts, the disappearance of TUBO cells was so quick as to preclude insight into the pathological cellular events involved.

Gene gun DNA vaccination eradicates established $r$-p $185^{\text {neu }}$ carcinomas. To assess the curative potential of DNA gene gun vaccination, WT BALB/c mice bearing established 2-, 4-, and 6-mm mean diameter TUBO carcinomas received the two plasmids shots 7 days apart (Figure 2). The 2-mm mean diameter solid carcinomas were measurable between 8 and 12 days after the TUBO cell challenge. In all mice untreated (Figure 2, d-f) or shot with the empty vector (Figure 3a), masses grew progressively, giving rise to large, p185 $5^{\text {neu }}$-positive carcinomas with a moderate leukocyte infiltrate associated with a slight production of proinflammatory cytokines (Table 3 and Figure 3 , a, c, e, g, and i). By contrast, in mice shot with p185 plasmid tumor, masses grew for the first 20 days and then started to shrink, and no tumor was palpable 50 days after the first shot (Figures $2 a$ and $3 b$ ). A marked infiltration by several leukocyte populations (Table 3 and Figure $3 \mathrm{~h}$ ) expressing high levels of IL-1, IL-4, IL-6, and TGF- $\beta 1$ (Table 3 and

Table 2

Ability of 185 plasmid-vaccinated WT BALB/c mice with immunosuppression by mAb administration and immune-related gene KOs to reject a lethal TUBO challenge

\begin{tabular}{|c|c|c|c|c|c|}
\hline \multirow{3}{*}{$\begin{array}{l}\text { Mice } \\
\text { WT BALB/c }\end{array}$} & \multicolumn{2}{|c|}{ mAb immunosuppression } & \multirow{3}{*}{$\begin{array}{c}\text { p185 plasmid } \\
\text { vaccination } \\
-\end{array}$} & & \\
\hline & mAb specificity & Time of administration & & \multicolumn{2}{|c|}{$\begin{array}{l}\text { Tumor takes per } \\
\text { challenged mouse }\end{array}$} \\
\hline & none & - & & $15 / 15$ & $0 \%$ \\
\hline WT BALB/c & none & - & + & $0 / 15$ & $100 \%$ \\
\hline WT BALB/c & anti-CD4 mAb during immunization & + & $10 / 10$ & $0 \%$ & \\
\hline WT BALB/c & anti-CD4 mAb & after challenge & + & $0 / 10$ & $100 \%$ \\
\hline WT BALB/c & anti-CD8 mAb during immunization & + & $0 / 10$ & $100 \%$ & \\
\hline WT BALB/c & anti-CD8 mAb & after challenge & + & $5 / 10$ & $50 \%$ \\
\hline WT BALB/c & anti-PMN mAb during immunization & + & $0 / 8$ & $100 \%$ & \\
\hline WT BALB/c & anti-PMN mAb & after challenge & + & $8 / 8$ & $0 \%$ \\
\hline BALB- $\mu \lg K O$ & - & - & - & $5 / 5$ & $0 \%$ \\
\hline BALB- $\mu$ IgKO & - & - & + & $0 / 5$ & $100 \%$ \\
\hline BALB-FcyRI/IIKO & - & - & - & $8 / 8$ & $0 \%$ \\
\hline BALB-FcyRI/IIKO & - & - & + & $0 / 6$ & $100 \%$ \\
\hline BALB- $\beta 2 \mathrm{mKO}$ & - & - & - & $5 / 5$ & $0 \%$ \\
\hline BALB- $\beta 2 \mathrm{mKO}$ & - & - & + & $0 / 6$ & $100 \%$ \\
\hline BALB-CD1KO & - & - & - & $5 / 5$ & $0 \%$ \\
\hline BALB-CD1KO & - & - & + & $0 / 5$ & $100 \%$ \\
\hline BALB-MCP1KO & - & - & - & $5 / 5$ & $0 \%$ \\
\hline BALB-MCP1KO & - & - & + & $0 / 5$ & $100 \%$ \\
\hline BALB-IFN $\gamma \mathrm{KO}$ & - & - & - & $5 / 5$ & $0 \%$ \\
\hline BALB-IFN $\gamma K O$ & - & - & + & $0 / 5$ & $100 \%$ \\
\hline BALB-pfpKO & - & - & - & $5 / 5$ & $0 \%$ \\
\hline BALB-pfpKO & - & - & + & $0 / 5$ & $100 \%$ \\
\hline BALB-IFN $\gamma$-pfpKO & - & - & - & $5 / 5$ & $0 \%$ \\
\hline BALB-IFN $\gamma$-pfpKO & - & - & + & $5 / 5$ & $0 \%$ \\
\hline
\end{tabular}

Untreated WT BALB/c and WT BALB/c mice that received Ab 22, 18, 14, 8, and 5 days before challenge or 2, 5, 8, 11, and 13 after challenge and KO BALB/c mice were immunized with p 185 plasmid or empty vector 21 and 7 days before tumor challenge (day 0$)$. Tumor takes and percentage of protected mice were evaluated for 100 days after challenge. 


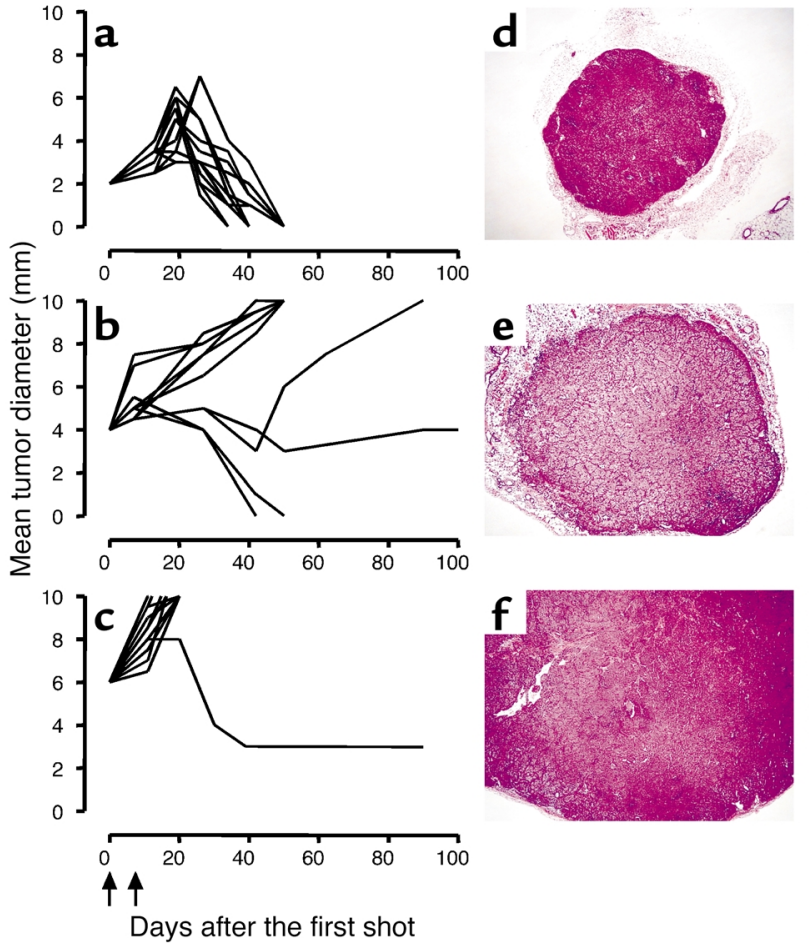

Figure 3j) was associated with ICAM-1, ELAM-1, and VCAM-1 expression by the activated endothelial cells of tumor microvessels. Residual neoplastic cells displayed low and cytoplasm-confined expression of r-p185 18 eu $($ Figure 3f). As the rejection progressed, an extremely marked fibrotic reaction led to complete disintegration and replacement of the tumor mass (Figure $3 \mathrm{~d}$ ).

Four-millimeter mean diameter TUBO tumors were evident between 16 and 19 days after challenge (Figure $2 \mathrm{e})$. In 4 of the 12 mice immunized with p 185 plasmids, tumors grew slowly for the first 20 days after vaccination, then tumors shrank rapidly in 3 mice and no tumor was palpable within 60 days after the first shot. In the fourth mouse, the tumor formed a stable mass about $4 \mathrm{~mm}$ in mean diameter that was diagnosed as a fibrotic scar deprived of neoplastic cells when it was

\section{Figure 3}

Morphological events associated with the eradication of the 2-mm mean diameter TUBO carcinomas in immunized WT BALB/c mice. Twenty days after the first shot with empty vector, a large, solid carcinoma (a) with a delicate stroma and without appreciable collagen deposition (c) formed by round, monomorphous, r-p185 18 neu-expressing neoplastic cells (e) was evident. Immunohistochemistry showed that the tumor mass was moderately infiltrated by PMNs (g) and characterized by a very slight production of the fibrogenic cytokine TGF- $\beta 1$ (i). At the same time, mice shot with $\mathrm{p} 185$ plasmids displayed small neoplastic cell aggregates (b), with an evident downmodulation of $185^{\text {neu }}$ expression on the cell membranes (f), surrounded by a massive collagen deposition and devastating fibrotic reaction evidenced as blue-green staining by the trichrome method (d). A marked infiltration by PMN (h) and expression of TGF- $\beta 1$ (j) were evident among and inside the neoplastic cell aggregates. Magnification, $\times 630$.

\section{Figure 2}

Ability of gene gun vaccination to cure established TUBO carcinomas. The left panels show growth and regression of 2-mm (a), 4-mm (b) or 6-mm (c) mean diameter carcinomas after two $\mathrm{p} 185$ plasmid shots (arrows). No modulation of tumor growth was observed after shots with empty plasmids (data not shown). The right panels show histological stages of the tumors. When the first shot was performed, the 2-mm carcinomas (d) consisted of well-defined neoplastic lobules surrounded by a delicate stroma. The 4-mm carcinomas (e) consisted of an established mass invading the subcutaneous fibroadipose tissue and almost completely occupying the microscope field. The 6-mm carcinomas ( $f$ ) could no longer be fully contained within the microscope field. These carcinomas show an expansive and invasive growth pattern. Magnification, $\times 25$.

pathologically examined 100 days after the first shot. In one mouse the tumor grew, shrank, and regrew,

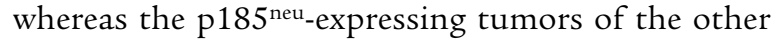
seven mice grew progressively (Figure $2 b$ ).

No evidence of a reduced growth rate was found for 11 of the 12 mice that received the first shot of $\mathrm{p} 185$ plasmid when the tumor was $6 \mathrm{~mm}$ in mean diameter, 25 days after challenge. Unfortunately, these mice had to be sacrificed for humane reasons within 16-25 days after the first shot, possibly before the immune reaction was fully established. In one mouse, the tumor

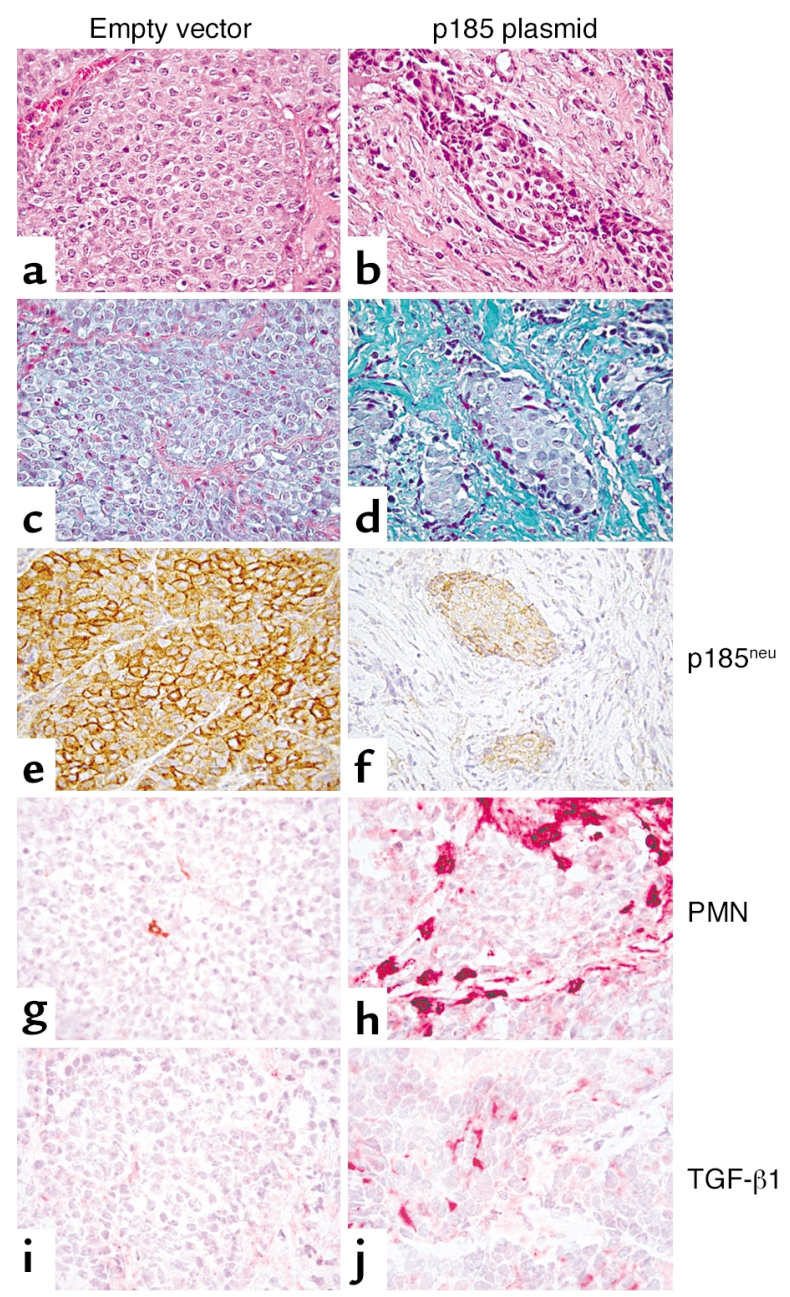




\section{Table 3}

Immunohistochemical analysis of TUBO tumor growth and rejection area in untreated or immunized WT BALB/c mice 20 days after the tumor reached $2 \mathrm{~mm}$ in mean diameter

$$
\begin{gathered}
\text { Untreated mice } \quad \begin{array}{c}
\text { Mice vaccinated } \\
\text { with p185 plasmid }
\end{array} \\
\text { with }
\end{gathered}
$$

\begin{tabular}{|c|c|c|}
\hline \multicolumn{3}{|l|}{ Reactive cells } \\
\hline Dendritic cells & ND & $4 \pm 1$ \\
\hline Macrophages & $18 \pm 4$ & $24 \pm 6$ \\
\hline PMNs & $7 \pm 2$ & $20 \pm 4$ \\
\hline CD8 lymphocytes & $6 \pm 2$ & $40 \pm 8$ \\
\hline CD4+ lymphocytes & ND & $30 \pm 6$ \\
\hline NK cells & $9 \pm 3$ & $20 \pm 4$ \\
\hline \multicolumn{3}{|c|}{ Endothelial adhesion molecules } \\
\hline ICAM-1 & + & ++ \\
\hline ELAM-1 & - & + \\
\hline VCAM-1 & - & + \\
\hline \multicolumn{3}{|l|}{ Cytokines } \\
\hline IL-1 $\beta$ & \pm & ++ \\
\hline TNF- $\alpha$ & \pm & + \\
\hline IFN- $\gamma$ & - & ++ \\
\hline $\mathrm{IL}-4$ & - & ++ \\
\hline IL-6 & \pm & ++ \\
\hline TGF- $\beta$ & - & ++ \\
\hline \multicolumn{3}{|c|}{$\begin{array}{l}\text { The vaccine was administered when tumors reached } 2 \mathrm{~mm} \text { in mean diameter } \\
\text { and } 7 \text { days later. Twenty days after the first shot, the reactive cells present in } \\
\text { the tumor were evaluated at } \times 400 \text { magnification in a } 0.180-\mathrm{mm}^{2} \text { field. At least } \\
\text { three samples (one sample per tumor growth area) and } 10 \text { randomly chosen } \\
\text { fields per sample were evaluated. Results are expressed as means } \pm \text { SD of pos- } \\
\text { itive cells per field evaluated on cryostat sections by immunohistochemistry. } \\
\text { The expression of endothelial adhesion molecules, cytokines, and chemokines } \\
\text { was defined as absent }(-) \text { or scarcely }( \pm) \text {, moderately }(+) \text {, or strongly }(++) \text { pres- } \\
\text { ent on cryostat sections stained with the Ab. WT BALB/c mice challenged with } \\
\text { p185 plasmid show significant protection as compared with untreated mice } \\
(P<0.005 \text {, two-tailed Student's } t \text { test). ND, not detected. }\end{array}$} \\
\hline
\end{tabular}

progressively regressed (Figure $2 \mathrm{c}$ ). The rejection of 4to 6-mm TUBO carcinomas was associated with a reaction similar to that described for 2-mm carcinomas.

Eradication of $r$-p185 nen carcinomas in immunodeficient mice. The 185 plasmid immunization by gene gun elicits an immunity sufficient to cure $2-\mathrm{mm}$ mean diameter tumors. The relative weight of immune mechanisms elicited was therefore reassessed in this more demanding situation. The first striking result was that the great majority of BALB- $\mu$ IgKO mice failed to be cured, even if immunization induced temporary tumor regression and delayed tumor growth (Figure 4a). The crucial role of antibody in r-p $185^{\text {neu }}$ tumor cure is supported by the inability of vaccination to cure BALB-Fc $\gamma R \mathrm{RI} / \mathrm{IIIKO}$ mice (Figure 4b). Poor effectiveness was also observed in BALB- $\beta 2 \mathrm{mKO}$ mice (Figure $4 \mathrm{~g}$ ). Although the pattern of tumor shrinkage in BALB-CD1KO (Figure 4h) and BALB-MCP1KO (Figure 4d) mice was not substantially different from that in WT BALB/c mice, tumors evaded the vaccine-induced immunity in a few mice from each of these groups, a feature never observed in WT BALB/c mice. Also, the great majority of BALB-IFN $\gamma \mathrm{KO}$ mice (Figure $4 \mathrm{e}$ ) were not cured, even if the vaccination markedly delayed tumor growth. Neither cure nor delayed tumor growth was evident for BALB-pfpKO (Figure 4f) or BALB-IFN $\gamma$-pfpKO (Figure 4c) mice.

Tumors progressively growing in the various groups of immunized KO mice consistently displayed high cellmembrane expression of $\mathrm{p} 185^{\text {neu }}$ and a reactive infiltrate lower than that of untreated WT BALB/c mice. Regressing tumors showed reactive infiltration and cytokine expression similar to those associated with tumors regressing in immunized WT BALB/c mice, even if these were sometimes weaker (data not shown).

\section{Discussion}

The surrogate model TAA addressed in these experiments (p185 neu) is the protein product of the Her-2/neu (Erb-B2 in humans) oncogene. This is a well-characterized signaling tyrosine kinase transmembrane receptor (52) whose overexpression in human carcinomas correlates with poor prognosis (53). Patients with Her-2/neuexpressing tumors may spontaneously develop anti$\mathrm{p} 185^{\mathrm{neu}}$ antibody and $\mathrm{T}$ cell responses (12), while the passive transfer of anti-p185 ${ }^{\text {neu }}$ monoclonal antibodies inhibits tumor growth and extends survival in a limited population of Her-2/neu patients (25). Because of the causal association of $\mathrm{p} 185^{\text {neu }}$ expression with tumor progression (52), $\mathrm{p}^{185^{\text {neu }}}$ is an attractive TAA for various forms of immunotherapy. In mice, r-p185 ${ }^{\text {neu }}$ is an interesting surrogate TAA, since it is a well-defined heterologous protein that differs from mouse p185neu in less than $6 \%$ of the amino acid residues (39). Despite this difference in the amino acid sequence between mouse and rat, the expression of $\mathrm{r}-\mathrm{p} 185^{\text {neu }}$ on the membrane of tumors transplanted in WT BALB/c mice does not elicit a detectable antibody and cellular response, nor does it impair their growth, moreover the reactive cell infiltrate is marginal and not higher than that commonly associated with syngeneic tumors ( 30 and present findings).

This apparent immunological invisibility of $\mathrm{r}-\mathrm{p} 185^{\text {neu }}$ may rest on the inability of tumor cells expressing r-p185 ${ }^{\text {neu }}$ to prime WT BALB/c T and B cells that recognize the xenogeneic r-p185 18 neu Presumably, rat epitopes of r-p185neu expressed on tumor cell membranes are insufficient to generate immunity in the absence of an effective cross-priming such as that which takes place after particle bombardment by gene gun. In effect, we observed that DNA vaccination of WT BALB/c mice with p185 plasmids administered by a gene gun not only elicited completely protective immunity against a subsequent challenge of r-p185 ${ }^{\text {neu}}-$ expressing TUBO cells, but the reaction triggered was sufficiently strong to eradicate palpable TUBO carcinomas.

Teasing out the mechanisms responsible for this protective immunity, we observed that the rejection of a tumor challenge by preimmunized mice had different requirements than the eradication of an established carcinoma. The induction of the anti-r-p185 ${ }^{\text {neu }}$ immune response rests on CD4 lymphocytes, whose removal during the immunization period abolished the induction of protective immunity. The cross-presentation by dendritic cells of antigens encoded by plasmids shot into the 

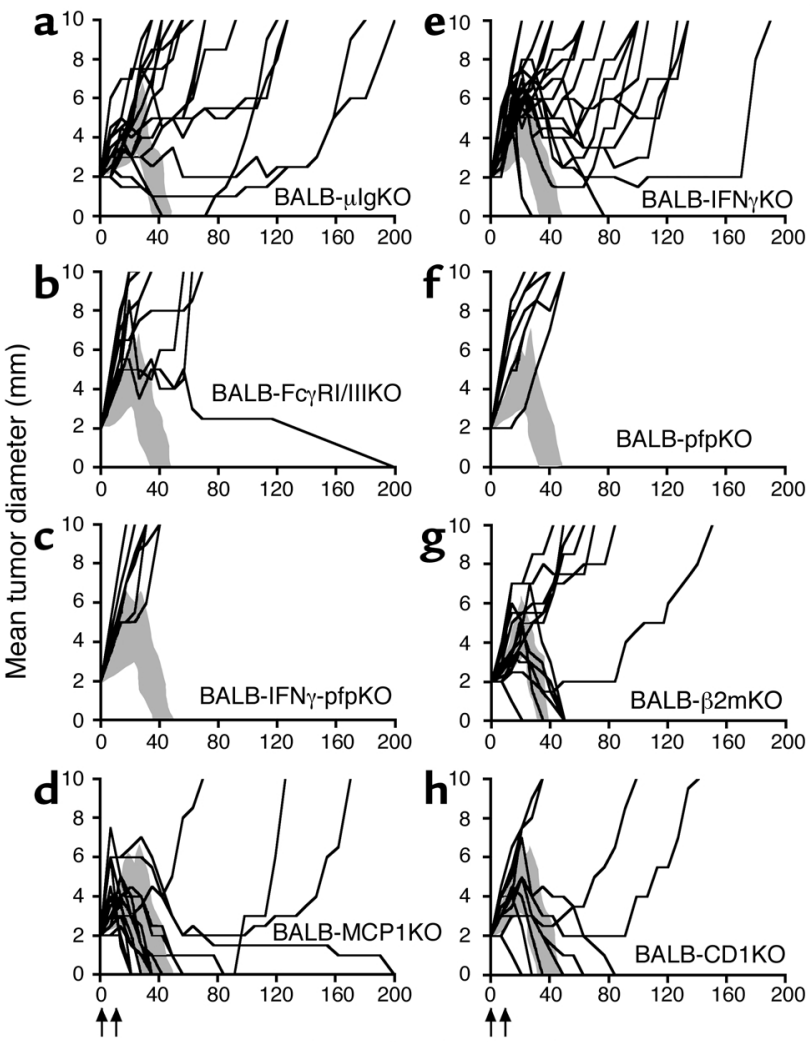

Days after the first shot

\section{Figure 4}

Mechanism of r-p185 carcinoma eradication. When the tumor was $2 \mathrm{~mm}$ in diameter, various $\mathrm{KO}$ mice were shot with p185 plasmid. Although all WT BALB/c mice were cured (20 of 20 cured at day $200)$ (Figure $2 \mathrm{a})$, vaccination did not cure any BALB-pfpKO mice $(0$ of 7 cured, $P<0.0001$ versus WT BALB/c) (f) or BALB-IFN $\gamma$-pfpKO mice ( 0 of 7 cured, $P<0.0001$ ) (c). Vaccination did not cure $89 \%$ of BALB- $\mu$ lgKO mice ( 2 of 18 cured, $P<0.0001$ ) (a), 88\% of BALBFcyRI/IIIKO mice ( 1 of 8 cured, $P<0.001$ ) (b), $86 \%$ of BALB-IFN $\gamma$ KO mice ( 3 of 21 cured, $P<0.0001)(\mathbf{e})$, and $78 \%$ of BALB- $\beta 2 \mathrm{mKO}$ mice (2 of 9 cured, $P<0.001)(\mathrm{g})$. Vaccination cured $54 \%$ of BALBCD1KO mice ( 6 of 11 cured, $P<0.006)(\mathbf{h})$ and $80 \%$ of BALBMCP1KO mice ( 12 of 15 cured, $P<0.07)($ d). Arrows show the days of the shots, and the gray area shows the growth and the rejection of the tumor in WT BALB/c mice bearing 2-mm tumors.

dermis accounts for this central role of $\mathrm{CD} 4 \mathrm{~T}$ cells in the induction of the protective response (40-43). Although the marked decrease of CD8 cells in BALB- $\beta 2 \mathrm{mKO}$ mice did not reduce protection, protection was noticeably reduced by the depletion of $\mathrm{CD} 8 \mathrm{~T}$ cells during the rejection of TUBO cells. BALB- $\beta 2 \mathrm{mKO}$ mice lack NK T cells and have desensitized NK cells, no FcyRs, and some small populations of CD8 T cells (45). NK T cells can suppress Th1 responses, so in their absence the response of the small $\mathrm{T}$ cell population might be more effective (54). By contrast, $\mathrm{BALB} / \mathrm{c}$ mice treated with antibodies to $\mathrm{CD} 8$ are devoid of CD8 T cells and probably also of CD8 DCs. In these immunized mice, the lack of resistance may directly result from the almost complete absence of $\mathrm{CD} 8 \mathrm{~T}$ cells. Alternatively, CD8 DCs may be required for an effective cross-presentation of $\mathrm{r}$-p185 $185^{\text {neu }}$ after gene gun vaccination. Surprisingly, protection afforded by vaccination was also abolished by depletion of PMNs. Indeed, pathological analysis of the TUBO cell rejection area supports a key role for both of these leukocyte populations, since they were very well represented and closely intermingled with damaged tumor cells. A similar critical role of PMNs was repeatedly observed in the cytokine-activated rejection of transplantable tumors $(51,55)$. Although the antitumor activity of the resting PMNs is marginal, it can be elicited and guided by cytokines released by $T$ cells (55), and the powerful destructive activity of PMNs can be guided by antibodies bound to Fc $\gamma R \mathrm{RI} / \mathrm{III}(20)$. However, the rejection of $\mathrm{p} 185^{\text {neu-express- }}$ ing TUBO cells by immunized mice took place even in the absence of antibodies (BALB- $\mu$ IgKO mice) (16), FcRmediated ADCC (BALB-Fc $\gamma$ RI/IIIKO mice) (44), NK cells that actively mediate ADCC (56), and NK T cells (47) associated with impaired macrophage function (57) (BALB-CD1KO mice). The rejection of the TUBO cell challenge even took place in the absence of pfp-mediated cytotoxicity (BALB-pfpKO mice) (49) and was not impaired by the absence of IFN- $\gamma$ (BALB-IFN $\gamma \mathrm{KO}$ mice) (46) or when macrophage recruitment was defective (BALB-MCP1KO mice) (48). Although numerous combinations of antibody depletion and gene KO may provide a more definite identification of the mechanisms involved, present data show that no single immune deficiency, besides PMNs, was crucial for the rejection of an $\mathrm{r}$-p185 $5^{\text {neu }}$ cell challenge. This finding suggests that the reaction elicited by $\mathrm{p} 185$ plasmids administered by gene gun was based on the induction of multiple mechanisms. In this way, a specific immune deficiency can be compensated by the numerous residual mechanisms. The complete abrogation of protection observed when two gene deficiencies (IFN- $\gamma$ and pfp) that do not individually impair the challenge rejection are combined (BALB-IFN $\gamma$ pfpKO mice) endorses this compensatory interpretation.

When the role of the distinct immune mechanisms was assessed in the eradication of an established and actively growing carcinoma, the results were diametrically different, since all the mechanisms assayed played a more or less essential role. Although immunization cured all WT BALB/c mice bearing a 2-mm carcinoma, almost no cure was observed in the absence of antibody, and none at all was observed in Fc $\gamma \mathrm{RI} / \mathrm{IIIKO}$ mice. Therefore, antibody and presumably also ADCC are prerequisites for tumor cure. The absence of pfp-dependent lytic mechanisms, an impairment in CD8 effector lymphocytes (BALB- $\beta 2 \mathrm{mKO}$ mice), and the decrease or absence of NK T cells (BALB- $\beta 2 \mathrm{mKO}$ mice and CD1KO mice) either abolished or strongly reduced the ability of immunized mice to eradicate the carcinoma. Carcinoma cure was also dramatically impaired in the absence of IFN- $\gamma$ (BALB-IFN $\gamma \mathrm{KO}$ mice). Even if the defective macrophage recruitment in BALB-MCP1KO mice poorly affected the ability to cure, carcinoma grew in $20 \%$ of these mice. The ability to generate an effective antitumor attack that overcomes the kinetics of tumor proliferation and tumor escape mechanisms appears to result from the 
combination of multiple immune mechanisms, each of which is necessary for efficient protection.

Anti-p185 $5^{\text {neu }}$ antibodies appear to have a differential role in the protection against $\mathrm{p} 185^{\text {neu }}$ tumors depending on the context in which their role is evaluated. In the absence of anti-p185 ${ }^{\text {neu }}$ antibodies, other pre-established reaction mechanisms protect against a lethal tumor challenge (35-38), whereas in a situation in which a rising immunity had to deal with the eradication of a preexisting tumor, their role became critical (34). These antibodies can directly impair the proliferation kinetics of p185 ${ }^{\text {neu-expressing tumors }}$ by interfering with the growth-signaling function of the $\mathrm{p} 185^{\text {neu }}$ receptor expressed on the tumor cell membrane (25-29). The marked membrane downmodulation of r-p185 18 and diminished nuclear positivity of proliferating cell nuclear antigen (PCNA) characterizing the cure of TUBO carcinomas points to a direct inhibitory activity, since reduced r-p185 ${ }^{\text {neu }}$ expression was sufficient for the impairment of tumor growth ability in vivo $(27,28,30,32)$. However, observations of pathology highlighted a massive PMN and NK infiltration during carcinoma eradication. In the presence of the high titer of anti-r-p185 18 antibodies elicited by $\mathrm{p} 185$ plasmid immunization, PMNs and NK cells may be activated to mediate ADCC $(20,58)$. The central role played by cells expressing Fc $\gamma \mathrm{RI} / \mathrm{III}$ and by PMNs strongly support this concept. The number of PMNs and NK cells - and, less markedly, that of macrophages infiltrating the tumor was significantly lower in carcinomas that continue to grow than in those that did regress. The mechanisms that regulate the tumor recruitment and local activation of these nonspecific effectors $(51,55)$ may play a significant role in deciding the growth or rejection of established carcinomas in immunized mice.

The massive cytokine expression in the tumor rejection area may explain not only the intratumoral recruitment of nonspecific and specific reactive cells but also the devastating fibrotic reaction that accompanied tumor regression. In fact, in addition to a high expression of TGF- $\beta 1$, a well known collagen-inducing mediator, other cytokines such as IL- $1 \beta$ and IL- 4 , which promote fibroblast proliferation and collagen deposition, are widely produced during the rejection process (58).

In conclusion, present data suggest that a lethal challenge by r-p185 $5^{\text {neu }}$-positive carcinoma cells can be completely rejected by preimmunized mice, not only in the absence of antibodies and Fc $\mathrm{RI} / \mathrm{III}$-positive cells but also in the absence of pfp-dependent cytotoxicity. It can be significantly inhibited even in the absence of CD4 and CD8 $\mathrm{T}$ effector cells. This rejection pattern suggests that several immune mechanisms can independently lead to tumor challenge rejection. However, when the induction of the immune response has to cope with the proliferative kinetics of a palpable tumor, efficient tumor eradication only results from the cooperation of these various mechanisms. Not only do antibodies, FcyRI/III-positive cells, IFN- $\gamma$, and pfp play a critical role, but complete protection also requires NK T cells and efficient macrophage recruitment. Although it is morphologically well docu- mented that a multicell-mediated reaction is associated with tumor rejection $(51,59)$, we have been able to show here the importance of each component in the process. The additional effect resulting from this cooperation implies that an antitumor vaccine able to trigger a multifaceted reaction, and not only a CTL or antibody response, could be particularly effective. In this case, the choice of the target TAA is critically important. $\mathrm{p} 185^{\text {neu }}$ is one of many tyrosine kinase receptors directly involved in cell growth and carcinogenesis that are expressed on the cell surface (52). Those TAAs that cannot be lost or downmodulated without impairing the cell oncogenic potential appear to be suitable targets for both $\mathrm{T}$ cell- and antibody-orchestrated immune reactivity (60).

With the use of a xenogeneic surrogate TAA, a few of the multiple and sometimes redundant mechanisms required for the immune eradication of tumors expressing membrane oncogenic tyrosine kinase receptors have been teased apart. However, the use of r-p $185^{\text {neu }}$ as an antigenic target in a mouse system makes a direct assessment of the relevance of these data to an active immunization against self-p185 $5^{\text {neu }}$ problematic. After gene gun immunization against the xenogeneic $\mathrm{p} 185^{\text {neu, }}$ the amino acid sequences that differ between rat and mouse can provide various types of $\mathrm{T}$ cell help and thus allow the elicitation of an effective immune response characterized by high-intensity and high-affinity effector mechanisms. However, gene gun vaccination may not be equally suitable for breaking down tolerance and inducing an effective immune response in the case of human $185^{\text {neu }}$, since it is a self-tolerated antigen widely expressed at low levels in multiple tissues.

\section{Acknowledgments}

The present work was made possible by the generous gift of BALB- $\mu \mathrm{IgKO}$ mice from Thomas Blankenstein (Free University, Berlin, Germany), BALB-CD1KO mice from Luc Van Kaer (Vanderbilt University, Nashville, Tennessee, USA), and BALB-MCP1KO mice from Barrett Rollins (Dana Farber Institute, Boston, Massachusetts, USA). We also thank Susanna Massasso for her skilled technical assistance and Simon West for editing the manuscript. This work was supported by grants from the Italian Association for Cancer Research (AIRC) and the Italian Ministry for University and Scientific and Technological Research (MURST; University of Turin). C. Curcio is a recipient of a University of Turin Fondazione Bossolasco fellowship. M.J. Smyth was supported by the National Health and Medical Research Council of Australia.

\footnotetext{
1. Boon, T., Cerottini, J.-C., Van den Eynde, B., Van der Bruggen, P., and Van Pel, A. 1994. Tumor antigens recognized by T lymphocytes. Annu. Rev. Immunol. 12:337-365.

2. Tureci, O., Sahin, U., and Pfreundschuh, M. 1997. Serological analysis of human tumor antigens: molecular definition and implications. Mol. Med. Today. 3:342-349.

3. Hanson, H.L., et al. 2000. Eradication of established tumors by CD8+ T cell adoptive immunotherapy. Immunity. 13:265-276.

4. Ward, P.L., Koeppen, H.K., Hurteau, T., Rowley, D.A., and Schreiber, H. 1990. MHC Class I and unique antigen expression by tumors that escaped from CD8+ $\mathrm{T}$ cell dependent surveillance. Cancer Res. 50:3851-3858.

5. Greenberg, P.D., Cheever, M.A., and Fefer, A. 1981. Eradication of
} 
disseminated murine leukemia by chemoimmunotherapy with cyclophosphamide and adoptively transferred immune syngeneic Lyt-1+2- lymphocytes. J. Exp. Med. 154:952-963.

6. Pericle, F., et al. 1994. An efficient Th-2-type memory follows CD8+ lymphocyte driven and eosinophil mediated rejection of a spontaneous mouse mammary adenocarcinoma engineered to release IL-4. J. Immunol. 153:5659-5673.

7. Forni, G., and Giovarelli, M. 1984. In vitro reeducated Thelper cells from sarcoma bearing mice inhibit sarcoma growth in vivo. J. Immunol. 132:527-533.

8. Forni, G., et al. 1988. Helper strategy in tumor immunology: expansion of helper lymphocytes and utilization of helper lymphokines for experimental and clinical immunotherapy. Cancer Metastasis Rev. 7:289-309.

9. Houghton, A.N., and Lloyd, K.O. 1998. Stuck in the MUC on the long and winding road. Nat. Med. 4:270-271.

10. Hellstrom, K.E., and Hellstrom, I. 1974. Lymphocyte-mediated cytotoxicity and blocking serum activity to tumor antigens. Adv. Immunol. 18:209-277.

11. Monach, P.A., Schreiber, H., and Rowley, D.A. 1993. CD4+ and B lymphocytes in transplantation immunity. II. Augmented rejection of tumor allografts by mice lacking B cells. Transplantation. 55:1356-1361.

12. Disis, M.L., et al. 1994. Existent T-cell and antibody immunity to HER-2/neu protein in patients with breast cancer. Cancer Res. 54:16-20.

13. Stockert, E., et al. 1998. A survey of the humoral immune response of cancer patients to a panel of human tumor antigens. J. Exp. Med. 187:1163-1167.

14. Jager, E., et al. 1999. Humoral immune responses of cancer patients against "Cancer-Testis" antigen NY-ESO-1: correlation with clinical events. Int. J. Cancer. 84:506-510.

15. Houbiers, J.G., et al. 1995. Antibodies against p53 are associated with poor prognosis of colorectal cancer. Br. J. Cancer. 72:637-641.

16. Qin, Z., et al. 1998. B cells inhibit the induction of T cell-dependent tumor immunity. Nat. Med. 4:627-630.

17. Dhodapkar, K.M., Krasovsky, J., Williamson, B., and Dhodapkar, M.V. 2002. Antitumor monoclonal antibodies enhance cross-presentation of cellular antigens and the generation of myeloma-specific killer T cells by dendritic cells. J. Exp. Med. 195:125-133.

18. Rafiq, K., Bergtold, A., and Clynes, R. 2002. Immune complex-mediated antigen presentation induces tumor immunity. J. Clin. Invest. 110:71-79. doi:10.1172/JCI200215640.

19. Vasovic, L.V., Dyall, R., Clynes, R.A., Ravetch, J.V., and Nikolic-Zugic, J. 1997. Synergy between an antibody and CD8+ cells in eliminating an established tumor. Eur. J. Immunol. 27:374-382.

20. Stockmeyer, B., et al. 1997. Preclinical studies with $\mathrm{Fc}$ (gamma)R bispecific antibodies and granulocyte colony-stimulating factor-primed neutrophils as effector cells against HER-2/neu overexpressing breast cancer. Cancer Res. 57:696-701.

21. Clynes, R.A., Towers, T.L., Presta, L.G., and Ravetch, J.V. 2000. Inhibitory Fc receptors modulate in vivo cytoxicity against tumor targets. Nat. Med. 6:443-446.

22. Mitchell, M.S. 2002. Cancer vaccines, a critical review - Part II. Curr. Opin. Investig. Drugs. 3:150-158.

23. DiFronzo, L.A., et al. 2002. Enhanced humoral immune response correlates with improved disease-free and overall survival in American Joint Committee on Cancer Stage II Melanoma patients receiving adjuvant polyvalent vaccine. J. Clin. Oncol. 20:3242-3248.

24. Riethmuller, G., et al. 1998. Monoclonal antibody therapy for resected Dukes C colorectal cancer: seven-year outcome of a multicenter randomized trial. J. Clin. Oncol. 16:1788-1794.

25. Pegram, M.D., and Slamon, D.J. 1999. Combination therapy with trastuzumab (Herceptin) and cisplatin for chemoresistant metastatic breast cancer: evidence for receptor-enhanced chemosensitivity. Semin. Oncol. 26:89-95.

26. Klapper, N.L., et al. 1997. A subclass of tumor-inhibitory monoclonal antibodies to ErbB-2/HER2 blocks crosstalk with growth factor receptors. Oncogene. 14:2099-2109.

27. Katsumata, M., et al. 1995. Prevention of breast tumour development in vivo by downregulation of the p185neu receptor. Nat. Med. 1:644-648.

28. Drebin, J.A., Link, V.C., Stern, D.F., Weinberg, R.A., and Greene, M.I. 1985. Down-modulation of an oncogene protein product and reversion of the transformed phenotype by monoclonal antibodies. Cell. 41:697-706.

29. Nanni, P., et al. 2000. p185neu protein is required for tumor and anchorage independent growth, not for cell proliferation of transgenic mammary carcinoma. Int. J. Cancer. 87:186-194.

30. Rovero, S., et al. 2000. DNA vaccination against rat Her-2/neu p185 more effectively inhibits carcinogenesis than transplantable carcinomas in transgenic BALB/c mice. J. Immunol. 165:5133-5142.

31. Dakappagari, N.K., Douglas, D.B., Triozzi, P.L., Stevens, V.C., and Kaumaya, P.T. 2000. Prevention of mammary tumors with a chimeric HER-2 B-cell epitope peptide vaccine. Cancer Res. 60:3782-3789.

32. Nanni, P., et al. 2001. Combined allogeneic tumor cell vaccination and systemic interleukin 12 prevents mammary carcinogenesis in HER-2/ neu transgenic mice. J. Exp. Med. 194:1195-1205.

33. Drebin, J.A, Link, V.C., and Greene, M.I. 1988. Monoclonal antibodies specific for the neu oncogene product directly mediate anti-tumor effects in vivo. Oncogene. 2:387-394.

34. Reilly, R.T., et al. 2001. The collaboration of both humoral and cellular HER-2/neu-targeted immune responses is required for the complete eradication of HER-2/neu-expressing tumors. Cancer Res. 61:880-883.

35. Chen, Y., Hu, D., Eling, D.J., Robbins, J., and Kipps, T. 1998. DNA vaccines encoding full-length or truncated Neu induce protective immunity against Neu-expressing mammary tumors. Cancer Res. 58:1965-1971.

36. Mukai, K., et al. 2002. HER2 peptide-specific CD8(+) T cells are proportionally detectable long after multiple DNA vaccinations. Gene Ther 9:879-888.

37. Foy, T.M., et al. 2001. Vaccination with Her-2/neu DNA or protein subunits protects against growth of a Her-2/neu-expressing murine tumor. Vaccine. 19:2598-2606

38. Pilon, S.A., Piechocki, M.P., and Wei, W.Z. 2001. Vaccination with cytoplasmic ErbB-2 DNA protects mice from mammary tumor growth without anti-ErbB-2 antibody. J. Immunol. 167:3201-3206.

39. Nagata, Y., et al. 1997. Peptides derived from a wild-type murine protooncogene c-erbB-2/HER2/2 neu can induce CTL and tumor suppression in syngeneic hosts. J. Immunol. 159:1336-1343.

40. Mahvi, D.M., Sheehy, M.J., and Yang, N.-S. 1997. DNA cancer vaccines: a gene gun approach. Immunol. Cell Biol. 75:456-460

41. Chen, C.-H., Suh, K.W., Choti, M.A., Pardoll, M., and Wu, T.-C. 1999. Gene gun-mediated DNA vaccination induces antitumor immunity against human papillomavirus type 16 E7-expressing murine tumor metastases in the liver and lungs. Gene Ther. 6:1972-1981.

42. Feltquate, D.M., Heaney, S., Webster, G.R., and Robinson, H.L. 1997. Different $\mathrm{T}$ helper cell types and antibody isotypes generated by saline and gene gun DNA immunization. J. Immunol. 158:2278-2284.

43. Porgador, A., et al. 1998. Predominant role for directly transfected dendritic cells in antigen presentation to CD8 T cells after gene gun immunization. J. Exp. Med. 188:1075-1082.

44. Clynes, R., Takechi, Y., Moroi, Y., Houghton, A., and Ravetch, J. 1998. Fc receptors are required in passive and active immunity to melanoma. Proc. Natl. Acad. Sci. U. S. A. 95:652-656.

45. Epstein, S.L., et al. 2000. Vaccination with DNA encoding internal proteins of influenza virus does not require $\mathrm{CD} 8(+)$ cytotoxic lymphocytes: either CD4 (+) or CD8 (+) T cells can promote survival and recovery after challenge. Int. Immunol. 12:91-101.

46. Cantin, E., Tanamachi, B., Openshaw, H., Mann, J., and Clarke, K. 1999. Gamma interferon (IFN-gamma) receptor null-mutant mice are more susceptible to herpes simplex virus type 1 infection than IFN-gamma ligand null mutant mice. J. Virol. 73:5196-5200.

47. Mendiratta, S.V., et al. 1997. CD1d1 mutant mice are deficient in natural $\mathrm{T}$ cells that promptly produce IL-4. Immunity. 6:469-477.

48. Huang, D., Wang, J., Kivisakk, P., Rollins, B., and Ransohoff, R.M. 2001. Absence of monocyte chemoattractant protein 1 in mice leads to decreased local macrophage recruitment and antigen specific $\mathrm{T}$ helper cell type 1 immune response in experimental autoimmune encephalomyelitis. J. Exp. Med. 193:713-725.

49. Smyth, M.J., et al. 1999. Perforin is a major contributor to NK cell control of tumor metastasis. J. Immunol. 162:6658-6662.

50. Paglia, P., Chiodoni, C., Rodolfo, M., and Colombo, M.P. 1996. Murine dendritic cells loaded in vitro with soluble protein prime cytotoxic $\mathrm{T}$ lymphocytes against tumor antigen in vivo. J. Exp. Med. 183:317-322.

51. Musiani, P., et al. 1997. Cytokines, tumour-cell death and immunogenicity: a question of choice. Immunol. Today. 18:32-36.

52. Yarden, Y., and Sliwkowski, M.X. 2001. Untangling the ErbB signaling network. Nat. Rev. Mol. Cell. Biol. 2:127-136.

53. Gullick, W.J., et al. 1991. C-erbB-2 protein overexpression in breast cancer is a risk factor in patients with involved and uninvolved lymph nodes. Br. J. Cancer. 63:434-438.

54. Terabe, M., et al. 2000. NKT cell-mediated repression of tumor immunosurveillance by IL-13 and IL-4R-STAT6 pathway. Nat. Immunol. 1:515-520.

55. DiCarlo, E., et al. 2001. The intriguing role of polymorphonuclear neutrophils in antitumor reactions. Blood. 97:339-345.

56. Santoni, A., Herberman, R.B., and Holden, H.T. 1979. Correlation between natural and antibody-dependent cell-mediated cytotoxicity against tumor targets in the mouse. II. Characterization of the effector cells. J. Natl. Cancer Inst. 63:995-1003.

57. Nieuwenhuis, E.E., et al. 2002. CD1d-dependent macrophage-mediated clearance of Pseudomonas aeruginosa from lung. Nat. Med. 8:588-593.

58. Horst Ibelgaufts' Cytokines Online Path Finder Encyclopedia. http://www.copewithcytokines.de.

59. Rodolfo, M., et al. 1998. IgG2a induced by IL-12-producing tumor cell vaccines but not IgG1 induced by IL-4 vaccine are associated with the eradication of experimental metastases. Cancer Res. 58: 5812-5817.

60. Lollini, P.-L., and Forni, G. 2002. Antitumor vaccines: is it possible to prevent a tumor? Cancer Immunol. Immunother. 51:409-416. 